\title{
Continuity of Care Record (CCR) Action Plan
}

Lantana Consulting Group 


\title{
Continuity of Care Record (CCR) Action Plan
}

\author{
Prepared for \\ National Institute of Standards and Technology \\ Gaithersburg Md 20899-8202
}

By

Lantana Consulting Group

June 2011

U.S. Department of Commerce

Rebecca M. Blank, Acting Secretary

National Institute of Standards and Technology Patrick D. Gallagher, Under Secretary for Standards and Technology and Director

Certain commercial entities, equipment, or materials may be identified in this document in order to describe an experimental procedure or concept adequately. Such identification is not intended to imply recommendation or endorsement by the National Institute of Standards and Technology, nor is it intended to imply that the entities, materials, or equipment are necessarily the best available for the purpose. 


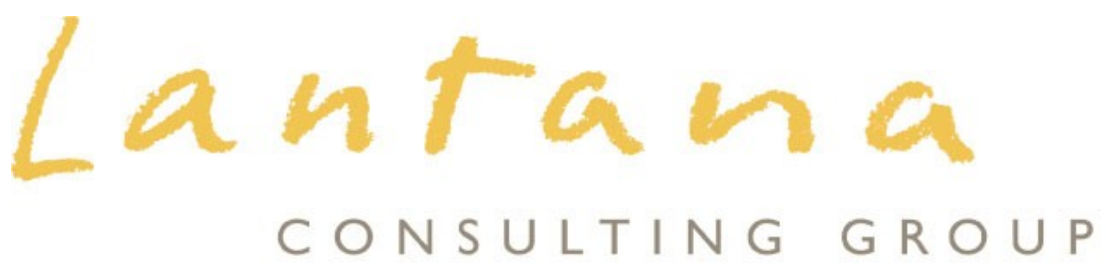

\section{Continuity of Care Record (CCR) Action Plan}

June 2011

\section{Prepared by Lantana Consulting Group}

for

National Institute of Standards and Technology

Lantana Consulting Group

Prepared for NIST

ü 2011, all rights reserved
CCR Standard Action Plan June 2011

Page 3 
(C) 2011 Lantana Consulting Group All rights reserved.

\section{Lantana Consulting Group}

PO Box 177

East Thetford, VT 05043

www.lantanagroup.com

Liora Alschuler

Chief Executive Officer

liora.alschuler@lantanagroup.com

Bob Dolin, MD, FACP

President and Chief Medical Officer

bob.dolin@lantanagroup.com

Lauren Wood, Ph.D.

Senior Project Manager

lauren.wood@lantanagroup.com

Gaye Dolin, RN, MSN

Senior Nurse Informaticist

gaye.dolin@,lantanagroup.com

Jingdong Li, MD

Chief Architect

jingdong.li@lantanagroup.com

Yan Heras

Senior Medical Informaticist

yan.heras@lantanagroup.com

Susan Hardy

Senior Technical Editor

susan.hardy@lantangroup.com

Lantana Consulting Group

Prepared for NIST

ü 2011, all rights reserved
CCR Standard Action Plan June 2011

Page 4 


\section{Table of Contents}

Executive Summary 4
Introduction 5
Recommended Actions 6
Refining the Standard 6
Profiling the Standard7
Testing and Validation
Error Handling 8
Certifying Developers and Architects 8
Creating Additional Documentation 8

Conclusions 9

References $\quad 10$

Acronyms and Abbreviations 11

Lantana Consulting Group CCR Standard Action Plan June 2011

Prepared for NIST Page 5

ü 2011, all rights reserved 
Executive Summary

This document is the action plan to improve the suitability of ASTM Continuity of Care Record (CCR) to support the Department of Health and Human Services' Stage 1 Meaningful Use. Lantana Consulting Group prepared this report for the National Institute of Standards and Technology (NIST) to accompany the related "CCR Suitability Analysis".

Based on our analysis, we believe that the CCR can be made suitable for Meaningful Use if the action described in this plan are carried out. We recommend six categories of actions that correspond to the issues identified in the Suitability Analysis:

- Refine the standard to add constraint and extensibility mechanisms, develop templates, add a vocabulary binding syntax, and define testability.

- Profile the standard to define elements and vocabularies that are needed for Meaningful Use.

- Create more testing tools and a reference implementation to improve testing and validation.

- Improve the process for error handling.

- Create a certification program for developers and architects.

- Create additional documentation. 
Introduction

This document is the action plan to improve the suitability of ASTM Continuity of Care Record (CCR) to support the Department of Health and Human Services' Stage 1 Meaningful Use.

Lantana Consulting Group prepared this report for the National Institute of Standards and

Technology (NIST) to accompany the related "CCR Suitability Analysis".

This plan recommends actions for all issues identified in the "CCR Suitability Analysis". In this plan, we also consider the interdependence between the recommended actions. Resolution of these issues may encourage more widespread adoption of the CCR standard.

We do not duplicate our analysis in this document, but do refer to it wherever applicable. This report does not discuss the assignment of responsibility for managing the change process.

Any mention of commercial products or organizations in this report is for information only; it does not imply recommendation or endorsement by Lantana or NIST.

1 Both CCR reports were prepared under contract SB134110SE0881.

Lantana Consulting Group

Prepared for NIST

ü 2011, all rights reserved
CCR Standard Action Plan June 2011

Page 7 


\section{Recommended Actions}

We recommend six categories of actions: refining the standard, profiling the standard, testing and validation, error handling, certifying developers and architects, and creating additional documentation.

\section{Refining the Standard}

The CCR standard contains useful information and concepts. We believe the standard would be more flexible and easier to implement, and could more fully support the Meaningful Use requirements if the following modifications were made:

- Add a constraint mechanism to meet specific use cases, such as a Meaningful Use profile of CCR, as described in "Profiling the Standard". A constraint mechanism restricts the original standard to meet specific business and content needs.

- Develop templates from the data objects, fields, types, and groups that are frequently reused. The definition of each template should include what is allowed within it, the precise meaning of allowed sub-elements, and any constraints on its use. Developers could use these templates to create profiles of the CCR to meet different use cases; they could also reuse them in different document types.

- Add an extensibility mechanism to meet specific use cases. The extensibility mechanism defines a process for adding certain elements or structures. Implementers could use an extensibility mechanism to create a localized version of the CCR for a jurisdiction that requires information not currently allowed by the CCR standard.

- Define a vocabulary binding method. Vocabulary binding defines code and value sets for specified elements within the standard. The vocabulary binding method can be used in constraint definitions, in localization, and during validation.

- Refine the definitions, explanations, descriptions, requirements, and restrictions in the CCR standard to increase the testability of CCR documents. Each requirement and restriction should be written using a consistent style, and each should be individually testable unless it is based on an untestable real-world condition. Improved testability makes it easier to create test suites and testing tools. It also decreases any ambiguity in the CCR standard, making it easier to understand and ultimately easier to implement correctly. We discuss this further in the Testing and Validation section.

Several of these recommendations are needed for the creation of a CCR profile for Meaningful Use, which is described in the next section.

\section{Profiling the Standard}

A CCR profile is required to meet the Meaningful Use requirements. Using the aboverecommended refinements to the standard, this profile would

- Define the elements that are required for Meaningful Use and use the constraint mechanism to require them in the profile. Validation and test suites will then be able to test for the presence or absence of the required elements.

$\begin{array}{lll}\text { Lantana Consulting Group } & \text { CCR Standard Action Plan } & \text { June } 2011 \\ \text { Prepared for NIST } & \text { Page } 8 \\ \text { ü 2011, all rights reserved } & \end{array}$


- Use the extensibility mechanism to add selected Meaningful Use items. The definition of specific elements, such as the smoking status, makes it easier to aggregate information from different sources.

- Use the vocabulary binding method to constrain the vocabularies for Meaningful Use. Specify how to reference the relevant vocabularies, such as SNOMED CT (Systematized Nomenclature of Medicine, Clinical Terms), LOINC (Logical Observation Identifiers Names and Codes), RxNorm, and the National Health Interview Survey (NHIS) vocabulary for smoking status.

- Define how CCR Meaningful Use documents should be rendered ("rendering expectations"). A clear definition of what is expected when a CCR document is rendered ensures that information encoded by the originating clinician is shown to the reader. Because of the large variability in systems that could use the CCR, the defined expectations must be simple (for example "this information, if present, must be shown, and if not present, the words 'IS NOT DEFINED' must be shown").

\section{Testing and Validation}

A validator exists for the CCR standard, written as a J2EE servlet; it implements a large amount of the functionality expected in a test suite. The following actions would provide additional functionality:

- Add a test for rendering expectations, including any that may be part of a Meaningful Use profile. A minimum test would be to create sample files that trigger defined expectations and then examine the files visually to ensure all required information is rendered appropriately.

- Provide Schematron test files as well as the schema files for general automated testing.

- If a Meaningful Use profile is developed, create Schematron files to test compliance with the profile.

- Provide sample files that exercise various parts of the standard. Include samples for Meaningful Use and "bad" sample files that trigger defined Schematron warnings and errors.

- Create a thorough, documented reference implementation. The reference implementation can be used to test and validate instances and to show developers how to implement each feature. It can test profiles and constraint mechanisms where edge cases may be important.

\section{Error Handling}

Any standard has errors and ambiguities. In all cases, readily available, vendor-independent information on errata improves applications and implementations of CCR. Currently, there does not appear to be a publicly available archive of errors apart from some vendor-specific forums. We recommend the following actions:

- Create a vendor-independent process for handling errors that is readily available to developers and implementers.

- Create a vendor-independent, publicly available forum or archive system for reported errors or ambiguities.

Lantana Consulting Group

Prepared for NIST

ü 2011, all rights reserved
CCR Standard Action Plan June 2011

Page 9 
- Create a reference implementation to test for potential errors and to clarify the intent of the CCR standard in cases where the standard is ambiguous (see also "Testing and Validation").

\section{Certifying Developers and Architects}

We recommend the creation of a certification program for developers and architects that motivates them to gain in-depth proficiency in the CCR standard and its implementation.

\section{Creating Additional Documentation}

CCR documentation exists for both the standard itself and for vendor-specific implementations. The CCR standard is relatively simple for those who understand the concepts and, as such, does not require much extra documentation. Our recommendations for this category overlap with recommendations for other categories:

- Create forums where developers or implementers can discuss the standard, issues they have with it, or potential improvements. This could be located with a forum for discussing errors.

- Create a reference implementation.

- If a Meaningful Use profile of the CCR is created, create documentation specific to that profile. 


\section{Conclusions}

The CCR standard is widely implemented and is generally considered sufficient for ambulatory settings and to give patients access to their health information. We found, however, that the CCR standard does not satisfy many suitability requirements, including some required for Meaningful Use. We recommend a number of actions that, if implemented, will ensure that the CCR standard meets all criteria.

Chief among these actions are refining the standard to make it both extensible and subject to constraint to meet particular use cases. We recommend that a profile be created to meet the Meaningful Use requirements. The standard should be refined to increase its testability, which will also make it easier to understand and implement. Aids to testing, including Schematron files and a reference implementation, should be created to help clarify the intent of the standard. A certification program for developers and implementers would also be advantageous. Finally, there should be a publicly available, vendor-independent way for developers and implementers to find, report and review known errors and ambiguities.

A CCR standard that includes the results of these action items will be more suitable for its stated purpose as well as for Stage 1 Meaningful Use. 


\section{References}

- ASTM Standard E2369 - 05e1. Standard Specification for Continuity of Care Record (CCR). DOI: 10.1520/E2369-05E01. 2005. http://www.astm.org/Standards/E2369.htm

- Lantana Consulting Group. CCR Suitability Analysis, May 2011. Related document prepared for NIST.

Lantana Consulting Group

Prepared for NIST

ü 2011, all rights reserved
CCR Standard Action Plan June 2011 Page 12 


\section{Acronyms and Abbreviations}

ASTM Originally called the American Society for Testing and Materials

CCR Continuity of Care Record

LOINC Logical Observation Identifiers Names and Codes

NHIS National Health Interview Survey

NIST National Institute of Standards and Technology

SNOMED CT Systematized Nomenclature of Medicine, Clinical Terms

Lantana Consulting Group

Prepared for NIST

ü 2011, all rights reserved
CCR Standard Action Plan June 2011

Page 13 